\title{
Nuclear Magnetic Resonance Study of Dichloromethane Adsorbed by Poly( $\gamma$-benzyl-L-glutamate) Film
}

\author{
Toshifumi Hiraoki, Akihiro Tsutsumi, Kunio Hikichi, \\ and Motozo KANeKo \\ Department of Polymer Science, Faculty of Science, \\ Hokkaido University, Sapporo 060, Japan.
}

(Received January 6, 1975)

\begin{abstract}
The proton-magnetic-resonance study has been carried out on $\operatorname{poly}(\gamma$ benzyl-L-glutamate) (PBLG) film which absorbs dichloromethane $\left(\mathrm{CH}_{2} \mathrm{Cl}_{2}\right)$. Spectra consist of a central sharp doublet superimposed on a broad signal. Separation of the central doublet, which was assigned to adsorbed $\mathrm{CH}_{2} \mathrm{Cl}_{2}$, was found to depend on the angle between the film normal and the magnetic field. The angular dependence of the second moment of the doublet was interpreted in terms of an appropriate model for molecular orientation of $\mathrm{CH}_{2} \mathrm{Cl}_{2}$ with respect to the PBLG helix and for motion of $\mathrm{CH}_{2} \mathrm{Cl}_{2}$.

KEY WORDS NMR / Poly( $\gamma$-benzyl-L-glutamate) / Dichloromethane / Film / Doublet / Planar Orientation /
\end{abstract}

It is well known that concentrated solutions of poly( $\gamma$-benzyl-L-glutamate) (PBLG) dissolved in organic solvents such as dichloromethane, chloroform, dioxane, etc., are liquid crystalline. ${ }^{1}$ In such solvents the PBLG molecule has an $\alpha$ helical conformation and a rigid rod-like shape. In the liquid crystalline state, PBLG molecules are arranged parallel to each other, forming large bundles. NMR studies on dichloromethane and/or dibromomethane solutions of PBLG have shown that the spectra of solvents consist of a doublet due to the dipole-dipole interaction of two protons in solvent molecules. ${ }^{2-5}$ The separation of the doublet was found to show an angle dependence. The doublet in solution has been considered as arising from the orientation of the solvent molecules in the direction of the magnetic field which is induced by the orientation of the PBLG molecule.

In the solid film, the arrangement and packing of the helical PBLG molecules are quite similar to those in the liquid crystalline. ${ }^{6}$ The molecules are packed in a slightly distorted hexagonal array and are parallel to the film surface. ${ }^{6}$

Samulski and Tobolsky ${ }^{7}$ have observed splitting in NMR spectra of dichloromethane adsorbed by PBLG films. The splitting indicates the orientation of the adsorbed solvent molecules in the film, which is also believed to be caused by a special arrangement of host polymers. However, no detailed study has been reported. In this work, we carried out NMR measurements of dichloromethane $\left(\mathrm{CH}_{2} \mathrm{Cl}_{2}\right)$ adsorbed by the PBLG film as a function of the angle between the film and the magnetic field in order to examine the orientation of the adsorbed molecules relative to the PBLG helix and the arrangement of the latter in the film.

\section{EXPERIMENTAL}

PBLG used in this work was prepared by polymerization of $\mathrm{N}$-carboxyanhydride in dioxane with a trace of hexamethylenediamine as an initiator at room temperature. The molecular weight was estimated to be $20.0 \times 10^{4}$ from the intrinsic viscosity in $N, N$-dimethylformamide (DMF).

A film of PBLG was cast on a glass plate from concentrated chloroform solution (ca. 10 $\mathrm{w} / \mathrm{v} \%$ ) at room temperature. A piece of the film cut in a rectangular shape $(0.015 \mathrm{~g})$ was placed in an NMR sample tube and then dried in vacuo at a temperature of $110^{\circ} \mathrm{C}$ for a day to remove all traces of solvent. About $30 \mu \mathrm{l}$ of $\mathrm{CH}_{2} \mathrm{Cl}_{2}$ was introduced into the sample tube with care being taken to avoid direct contact with the PBLG sample. Immediately after the 


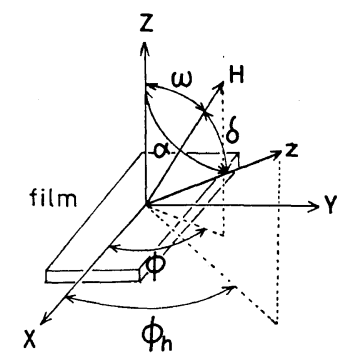

Figure 1. The coordinate system fixed in the film. The $Z$ axis is taken along the normal of the film plane. The $X Y$ plane is parallel to the film plane. The direction of the magnetic field $\boldsymbol{H}$ and the helical axis $z$ are specified by sets of angles $(\omega, \phi)$ and $\left(\alpha, \phi_{\mathrm{h}}\right)$, respectively, and $\delta$ is the angle between $\boldsymbol{H}$ and $z$.

introduction of $\mathrm{CH}_{2} \mathrm{Cl}_{2}$ the sample tube was sealed off. Spectral grade dichloromethane was used without further purification. NMR measurements were made after complete vaporization of $\mathrm{CH}_{2} \mathrm{Cl}_{2}$.

NMR spectra were recorded at various orientations of the sample at a temperature of $20^{\circ} \mathrm{C}$ using a JEOL JNS-H type (40 MHz) spectrometer.

The orientation of the sample is defined by the polar angle $\omega$ and the azimuthal angle $\phi$ as indicated in Figure 1, in which the coordinate system $X Y Z$ is fixed in the rectangular film, the $Z$ axis being normal to the film surface, the $X Y$ plane being in the film surface, and $\boldsymbol{H}$ the direction of the magnetic field.

\section{RESULTS AND DISCUSSION}

The spectrum consists of a central sharp signal superimposed on a broad one. The central singnal is assigned to $\mathrm{CH}_{2} \mathrm{Cl}_{2}$ protons, since its relative intensity increases with increasing amounts of adsorbed $\mathrm{CH}_{2} \mathrm{Cl}_{2}$. This signal was found to show a pronounced dependence on the angle $\omega$, but not on $\phi$.

In Figure 2, the central singnal is shown at various angles of $\omega$ and at an angle of $\phi=90^{\circ}$. A doblet signal is observed over the range of $\omega$ from $0^{\circ}-45^{\circ}$, where the separation of the doublet decreases with increasing $\omega$. At angles higher than $45^{\circ}$ a single broad line appears.

Figure 3 shows the second moment of the central signal plotted against $\omega$. The second

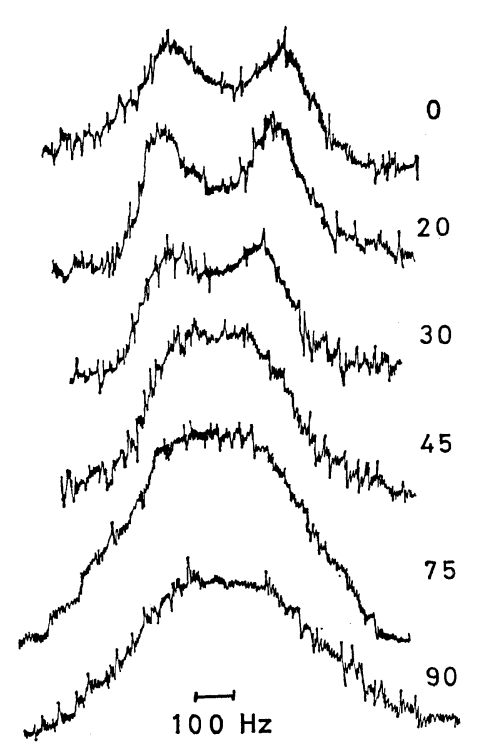

Figure 2. NMR spectra of adsorbed dichloromethane in the PBLG film taken at a temperature of $20^{\circ} \mathrm{C}$ and at an angle of $\phi=90^{\circ}$. The values of the angle $\omega$ are shown on the right-hand side of the spectra.

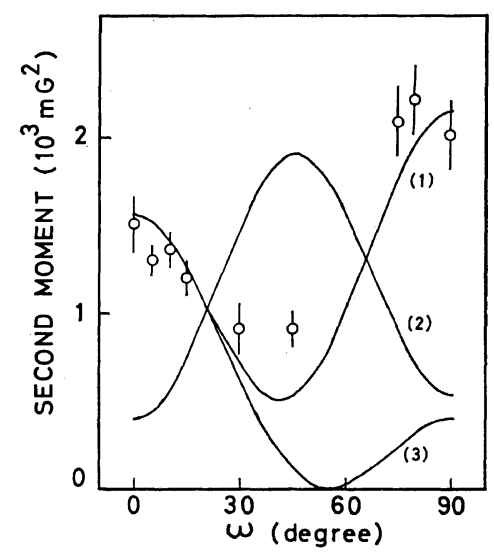

Figure 3. The observed and theoretical second moment $v s$. the angle $\omega$. The circles shows the observed results. The curves (1)-(3) show the theoretical results for $C_{\beta}, 1.1 \times 10^{-2}$ : (1) $\alpha, 90^{\circ}$; (2) $\alpha, 45^{\circ}$; (3) $\alpha, 0^{\circ}$, respectively; where curve (3) is the value on a reduced scale of $(1 / 4)$.

moment has a minimum at $\omega=45^{\circ}$, and two maxima at $\omega=0^{\circ}$ and $90^{\circ}$, the latter maximum being the greater.

The pronounce dependence of the second 
NMR of $\mathrm{CH}_{2} \mathrm{Cl}_{2}$ Adsorbed by PBLG Film

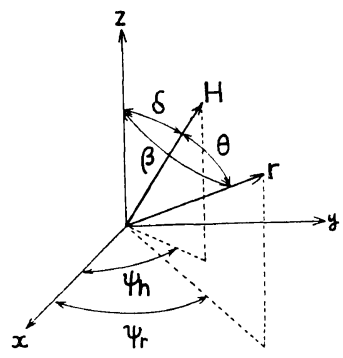

Figure 4. The coordinate system fixed in the PBLG helix. The $z$ axis is taken along the helical axis, and the $x y$ plane is perpendicular to the $z$ axis. The direction of the magnetic field $\boldsymbol{H}$ and the interproton vector $\boldsymbol{r}$ of $\mathrm{CH}_{2} \mathrm{Cl}_{2}$ are specified by sets of angles $\left(\delta, \phi_{\mathrm{h}}\right)$ and $\left(\beta, \phi_{\mathrm{r}}\right)$, respectively. $\theta$ is the angle between $\boldsymbol{H}$ and $\boldsymbol{r}$.

moment and the peak separation on $\omega$ indicates that adsorbed $\mathrm{CH}_{2} \mathrm{Cl}_{2}$ molecules are not free to reorient at random with respect to the film normal. To clarify this situation, the observed second moment was compared with the theoretical second moment.

NMR and dielectric studies have shown that the side chains of PBLG in the solid state undergo rapid motion at room temperature, whereas the helical backbone chains are presumably in the rigid state $^{8-11}$ Flory and Leonard ${ }^{12}$ have stated that adsorbed solvents enter in the first place into the side chains. Therefore, it seems appropriate for adsorbed $\mathrm{CH}_{2} \mathrm{Cl}_{2}$ to assume a motion relative to $\mathrm{PBLG}$ helices.

Figure 4 shows the geometrical arrangement of the magnetic field vector $\boldsymbol{H}$ and the interproton vector $\boldsymbol{r}$ for the theoretical calculation, in which the $z$ axis is the helical axis, and the $x y$ plane perpendicular to it. The second moment of a proton pair in rapid motion is given by $^{13}$

$$
\left\langle\Delta H^{2}\right\rangle=(3 / 4) \hbar^{2} \gamma^{2} I(I+1) r^{-6}\left(\left\langle 3 \cos ^{2} \theta-1\right\rangle\right)^{2}
$$

where $r$ is the interproton distance, $\hbar$ Planck's constant devided by $2 \pi, \gamma$ the gyromagnetic ratio, $I$ nuclear spin, and $\theta$ the angle between the direction of the magnetic field and the interproton vector. The angle brackets denote the time average over the rapid motion of the proton pair.

We assume that the motion of adsorbed solvent in the side-chain region is isotropic about the helix axis and all values of $\psi_{\mathrm{r}}$ defined in Figure 4 are plausible. However, the value of the term $\left(3 \cos ^{2} \beta-1\right)$ may not be averaged out, where the angle $\beta$ is also defined in Figure 4. This assumption may be justified by consideration of the helical form of PBLG and the rapid motion of surrounding side chain. Thus we have

$$
\left\langle 3 \cos ^{2} \theta-1\right\rangle=C_{\beta}\left(3 \cos ^{2} \delta-1\right)
$$

where $C_{\beta}$ stands for $(1 / 2)\left(3\left\langle\cos ^{2} \beta\right\rangle-1\right)$.

If the direction of the helix axis is given by the polar angle $\alpha$ and the azimuthal angle $\phi_{\mathrm{h}}$ in the coordinate system $X Y Z$ fixed to the film (Figure 1), we get

$$
\cos \delta=\cos \omega \cos \alpha+\sin \omega \sin \alpha \cos \left(\phi-\phi_{\mathrm{h}}\right)
$$

where $\omega, \alpha$, and $\phi$ are angles defined in Figure 1. Since the observed second moment is independent of $\phi$, the helical axis is distributed uniformly about the film normal. Therefore the second moment equation should be averaged over $\phi$. Then we obtain

$$
\left\langle\Delta H^{2}\right\rangle=K C_{\beta}{ }^{2}\left(A \cos ^{4} \omega+B \cos ^{2} \omega+C\right)
$$

where

$$
\begin{aligned}
& A=(9 / 8)\left(35 \cos ^{4} \alpha-30 \cos ^{2} \alpha+3\right) \\
& B=(-3 / 4)\left(45 \cos ^{4} \alpha-42 \cos ^{2} \alpha+5\right) \\
& C=(1 / 8)\left(27 \cos ^{4} \alpha-30 \cos ^{2} \alpha+11\right)
\end{aligned}
$$

and

$$
K=(3 / 4) \hbar^{2} \gamma^{2} I(I+1) r^{-6} .
$$

If we put $r=1.78 \AA$ as the interproton distance of $\mathrm{CH}_{2} \mathrm{Cl}_{2}, K=14.07\left(\mathrm{G}^{2}\right)$.

The calculated second moment curve shows a considerable dependence on $\alpha$ and $C_{\beta}$. Some of the results are shown in Figure 3. It is found that a curve for $\alpha=90^{\circ}$ and $C_{\beta}=1.1 \times 10^{-2}$ fits the observed data fairly well. $\alpha=90^{\circ}$ indicates that PBLG helices are arranged paralled to the film plane, i.e., uniplanar orientation.

X-ray studies for PBLG film show that PBLG helices are packed parallel to the film plane. ${ }^{14}$ The present NMR results of $\alpha=90^{\circ}$ are in good agreement with the $\mathrm{X}$-ray results.

The nonzero value of $C_{\beta}$ indicates that adsorbed $\mathrm{CH}_{2} \mathrm{Cl}_{2}$ molecules are not free to reorient at random, indicating a preferred orientation of the $\mathrm{CH}_{2} \mathrm{Cl}_{2}$ molecule with respect to the helical 
axis. Although details of the reorientation of $\mathrm{CH}_{2} \mathrm{Cl}_{2}$ molecules have not been clarified, one of the possibilities is that proposed by Sobajima ${ }^{2}$ in the case of the liquid-crystal of PBLG. In his model, the $\mathrm{CH}_{2} \mathrm{Cl}_{2}$ molecule rotates about the $C_{2}$ axis of which the direction fluctuates but is directed on average to the helix axis. Then, we obtain

$$
C_{\beta}=(-1 / 2)\left\langle\frac{3 \cos ^{2} \varepsilon-1}{2}\right\rangle=(-1 / 2) S
$$

where $\varepsilon$ is the angle between the molecular axis and the helix axis. In this case $S$ has the meaning of the time-averaged order parameter of the molecular axis of $\mathrm{CH}_{2} \mathrm{Cl}_{2}$ with respect to the helical axis. The value of $S$ of $2.1 \times 10^{-2}$ obtained in this work is not so much greater than reported values for $\mathrm{CH}_{2} \mathrm{Cl}_{2}$ in a PBLG liquid crystal $^{2}$ and for DMF in a polyglutamic acid liquid crystal ${ }^{15}$. This indicates that the motion of the $\mathrm{CH}_{2} \mathrm{Cl}_{2}$ adsorbed in the film is not greatly restricted compared with that in the liquid crystal.

\section{CONCLUSION}

Angular dependence of the NMR splitting of $\mathrm{CH}_{2} \mathrm{Cl}_{2}$ adsorbed in the PBLG film can be interpreted in terms of the dipole-dipole interaction of the proton pair in the $\mathrm{CH}_{2} \mathrm{Cl}_{2}$ molecule. A small but nonzero value of the order parameter shows that orientation of the adsorbed $\mathrm{CH}_{2} \mathrm{Cl}_{2}$ fluctuates rapidly owing to thermal motion, but is not completely random. It was found from the comparison of the order parameters that the reorientation of adsorbed $\mathrm{CH}_{2} \mathrm{Cl}_{2}$ in the film is not much different from that in the liquid crystal. NMR results show that $\alpha=90^{\circ}$, indicating that the PBLG helices are arranged preferentially parallel to the film plane.

Acknowledgment. The authors are grateful to Professor J. Noguchi of Hokkaido University and his colleagues for valuable advices in preparing samples. This work was supported by the Ministry of Education.

\section{REFERENCES}

1. C. Robinson, Tetrahedron, 13, 219 (1961).

2. S. Sobajima, J. Phys. Soc. Japan, 23, 1070 (1967).

3. E. T. Samulski and A. V. Tobolsky, Macromolecules, 1, 555 (1968).

4. M. Panar and W. D. Phillips, J. Amer. Chem. Soc., 90, 3880 (1968).

5. R. D. Owell and R. L. Vold, ibid., 93, 5335 (1971).

6. D. A. D. Parry and A. Elliott, J. Mol. Biol., 25, 1 (1967).

7. E. T. Samulski and A. V. Tobolsky, Mol. Cryst. Liq. Cryst., 7, 433 (1969).

8. K. Hikichi, J. Phys. Soc. Japan, 19, 2169 (1964).

9. A. Tsutsumi, Jap. J. Appl. Phys., 9, 1125 (1970).

10. K. Hikichi, K. Saito, M. Kaneko, and J. Furuichi, J. Phys. Soc. Japan, 19, 577 (1964).

11. A. Tsutsumi, K. Hikichi, T. Takahashi, Y. Yamashita, N. Matsushima, M. Kanke, and M. Kaneko, J. Macromol. Sci.-Phys., B8 (3-4), 413 (1973).

12. P. J. Flory and W. J. Leonard, Jr., J. Amer. Chem. Soc., 87, 2102 (1965).

13. H. S. Gutowsky and G. E. Pake, J. Chem. Phys., 18, 97 (1950).

14. A. J. McKinnon and A. V. Tobolsky, J. Phys. Chem., 70, 1453 (1966).

15. E. T. Samulski and H. J. C. Berendsen, J. Chem. Phys., 56, 3920 (1972). 